\title{
Objective assessment of dietary exposure and cardiometabolic risk in homeless adults
}

\author{
Rosalind Fallaize ${ }^{1,2}$, Vigil Moyo ${ }^{1}$, Jonathan Tammam ${ }^{3}$, John Draper ${ }^{4}$ and Julie A. Lovegrove ${ }^{2}$ \\ ${ }^{1}$ School of Life and Medical Science, University of Hertfordshire, Hatfield, AL10 9AB, UK, \\ ${ }^{2}$ Hugh Sinclair Unit of Human Nutrition, University of Reading, Reading RG6 6AP, UK, \\ ${ }^{3}$ Centre for Nutrition and Health, Oxford Brookes University, OX3 OBP and \\ ${ }^{4}$ Institute of Biological, Environmental and Rural Sciences, Aberystwyth University, Aberystwyth, SY23 3DA, UK
}

Homelessness is frequently associated with nutritionally imbalanced diets. Previously 24-hour recalls and food frequency questionnaires (FFQ) were used to retrospectively quantify nutritional intake in homeless adults, however these methods are prone to misreporting. Furthermore, memory recall may be confounded in the homeless community whereby greater incidences of reward mediated behaviour are reported ${ }^{(1)}$.

We aimed to pilot the use of dried blood spot (DBS) sampling to assess dietary exposure and cardiometabolic risk factors in homeless adults $(n=20)$ residing in hostel accommodation, and sex- and age-matched housed adults $(n=20)$ recruited from Reading, UK. Self-reported diet was determined using the EPIC-Norfolk FFQ, and diet quality via the Healthy Eating Index (HEI-2015). DBS were analysed for c-reactive protein (CRP), total cholesterol and fatty acids using LC-MS (Vitas AS, Norway). Vascular function was assessed using a portable digital-volume-pulse monitor (Mobil-O-Graph ${ }^{\circledR}$ ). The Health Science Engineering \& Technology ECDA at the University of Hertfordshire (aLMS/SF/UH/02498(1)), Research and Ethics Committee at the University of Reading (UREC 17/31) and The Salvation Army Ethics Committee granted ethical approval for the study, which was conducted according to the guidelines laid down in the Declaration of Helsinki.

Hostel residents were matched for age (mean 43years), sex (40\% females/group), body mass index (hostel, $24.8 \mathrm{~kg} / \mathrm{m} 2 ; \mathrm{housed}$, $24.7 \mathrm{~kg} / \mathrm{m} 2)$ and ethnicity. Compared with the housed group, hostel residents had a higher augmentation index $(\mathrm{p}=0.049)$ and QRISK-2 score $(\mathrm{p}=0.044)$. Diet quality was $49 \%$ higher $(22.8$ points $)$ in the housed group $(\mathrm{p}<0.001)$, as were the component scores for fruit, total vegetables, whole grains and dairy $(p ' s<0.05)$. Hostel residents had higher median DBS CRP $(p=0.002), C 14: 0$ and $\mathrm{C} 16: 0$ (p's < 0.05) than housed adults, whereas C17:0, a saturated fatty acid associated with dairy intake, was significantly lower $(\mathrm{p}=$ 0.004), compared with the housed group. For DBS unsaturated fatty acids, C16:1, C18:2, n-6 and C22:6, n-3 were all higher in the housed group (p's <0.05).

Hostel residents had lower diet quality and increased inflammation and vascular stiffness compared with age- and sex- matched housed groups. DBS sampling was feasible and provided valuable objective data which could support the implementation of dietary interventions in homeless communities.

\section{Acknowledgments}

The authors would like to thank the volunteers for their participation in the study and the organisations involved in recruitment.

\section{References}

1. Seale JV, Fallaize R \& Lovegrove JA (2016) Nutr Res Rev 29(2):1-9. 